\title{
An evolutionary catalogue of galactic post-AGB and related objects ${ }^{\star}$
}

\author{
R. Szczerba ${ }^{1}$, N. Siódmiak ${ }^{2}$, G. Stasińska ${ }^{3}$, and J. Borkowski ${ }^{1}$ \\ 1 N. Copernicus Astronomical Center, Rabiańska 8, 87-100 Toruń, Poland \\ e-mail: szczerba@ncac.torun.pl \\ 2 Space Telescope Science Institute, 3700 San Martin Drive, Baltimore, MD 21218, USA \\ ${ }^{3}$ LUTH, Observatoire de Meudon, 5 place Jules Janssen, 92195 Meudon Cedex, France
}

Received 28 December 2006 / Accepted 6 March 2007

\section{ABSTRACT}

\begin{abstract}
Aims. With the ongoing AKARI infrared sky survey, of much greater sensitivity than IRAS, a wealth of post-AGB objects may be discovered. It is thus time to organize our present knowledge of known post-AGB stars in the galaxy with a view to using it to search for new post-AGB objects among AKARI sources.

Methods. We searched the literature available on the NASA Astrophysics Data System up to 1 October 2006, and defined criteria for classifying sources into three categories: very likely, possible and disqualified post-AGB objects. The category of very likely postAGB objects is made up of several classes.

Results. We have created an evolutionary, on-line catalogue of Galactic post-AGB objects, to be referred to as the Torun catalogue of Galactic post-AGB and related objects. The present version of the catalogue contains 326 very likely, 107 possible and 64 disqualified objects. For the very likely post-AGB objects, the catalogue gives the available optical and infrared photometry, infrared spectroscopy and spectral types, and links to finding charts and bibliography.
\end{abstract}

Key words. stars: AGB and post-AGB - stars evolution - catalogs

\section{Introduction}

Once they have burnt up their central hydrogen and helium low- and intermediate-mass ascend the asymptotic giant branch (AGB), where they burn hydrogen and helium in thin shells surrounding the core. This phase, during which the atmosphere expands and cools down, is accompanied by intense mass loss $\left(10^{-7}-10^{-4} M_{\odot} \mathrm{yr}^{-1}\right)$. When the mass of the remaining H-rich envelope becomes smaller than $\sim 10^{-2} M_{\odot}$ (the exact value is very much a function of the initial mass, see, for example, Schönberner 1983), the envelope begins to shrink and the effective temperature starts to increase (Paczyński 1971). If the temperature increases on a timescale shorter than the dispersion time of the matter previously ejected by the star, a planetary nebula (PN) will appear, as the result of the ionization of the circumstellar shell and of the emission of optical line radiation. The intermediate phase between the end of the AGB phase and the PN phase is called the post-AGB phase. It was formerly also referred to as the proto-PN phase or the pre-PN phase (see, for example, Lo \& Bechis 1976; Likkel et al. 1987; Hrivnak et al. 1989; Geballe et al. 1992; Kwok 1993). We prefer the term post-AGB object ${ }^{1}$, since this also covers objects that have left the AGB stage but will evolve to the white dwarf stage without ever becoming a planetary nebula. The first review devoted to such objects was by Zuckerman(1978).

* A stable version of the catalogue is available at the CDS via anonymous ftp to cdsarc.u-strasbg.fr (130.79.128.5) or via http://cdsweb.u-strasbg.fr/cgi-bin/qcat?]/A+A/469/799

1 The term post-AGB star has also sometimes been used to denote very hot objects, e.g. PG 1159 stars, which are different objects from the ones considered in this paper and in our catalogue.
Important material on post-AGB objects can be found in the Conference Proceedings edited by Kwok \& Pottasch(1987), Preite-Martinez (1987), Schwarz (1993), Le Bertre et al. (1999), Szczerba \& Górny (2001), and in the book by Habing \& Olofsson (2004), as well as in the review papers by Kwok (1993) and van Winckel (2003).

Post-AGB stars are difficult - and often impossible - to detect in optical wavelengths, being strongly obscured by their circumstellar dusty envelopes. Infrared sky surveys such as the $2 \mu \mathrm{m}$ Sky Survey (Neugebauer \& Leighton 1969), the Air Force Infrared Sky Survey (Kleinmann et al.1981) and especially the InfraRed Astronomical Satellite (IRAS) survey (Beichman et al. 1988) were essential for the discoveries of post-AGB objects in the past. With the ongoing AKARI (Shibai 2004) infrared sky survey, of much greater sensitivity than IRAS, a wealth of post-AGB objects will be discovered. It is thus time to organize our present knowledge of post-AGB stars in our Galaxy into an evolutionary, on-line catalogue that can easily be updated by future discoveries.

A preliminary compilation for this catalogue was presented by Szczerba et al. (2001) and included about 220 objects. We have now scanned all the literature available on the NASA Astrophysics Data System (ADS) up to 1 October, 2006. In its present version, the catalogue, to be referred to as "the Torun catalogue of Galactic post-AGB and related objects", contains 326 very likely, 107 possible and 64 disqualified post-AGB objects. A subsample of the very likely objects was used in the work by Stasińska et al.(2006), which showed that post-AGB stars, as a class, are valuable testbeds for theories of AGB nucleosynthesis, and in the work by Siódmiak et al. (2007), devoted to the Hubble Space Telescope snapshot survey of 
post-AGB objects characterized by different physical and chemical properties.

\section{Classification criteria}

Post-AGB sources form a very heterogeneous group of stars. A most difficult task in creating this catalogue was to define which criteria the objects should satisfy in order to be said to be post-AGB stars. In general, the criteria are based on the central star properties (spectral type and luminosity class) and/or circumstellar envelope (disc?) properties (infrared excess, spectral dust and/or gas features). However, for many sources data exists only for one of those components. In some cases the star is not directly observable and the only available information is about the envelope. Such a situation is expected for higher mass sources where the timescales of stellar evolution are much shorter than the timescales of envelope dispersion into the interstellar medium. In other cases, there is some information on the central star (luminosity class I-III and appropriate spectral type) but no (or only a small) infrared (IR) excess is detected. Such a situation is typical for lower mass sources with very long timescales of stellar evolution.

In defining criteria, we have been inspired by the precepts introduced by Kwok (1993), Van Winckel (2003) and Waelkens $\&$ Waters (2004). We emphasize that with the established criteria we have tried to cover all stellar objects classified for various reasons as post-AGB ${ }^{2}$. The coolest post-AGB objects included in our catalogue have spectral types not later than $\mathrm{K}$, while the hottest have effective temperatures below $25000 \mathrm{~K}$ (see Szczerba et al. 2001). We count as disqualified post-AGB objects those that have central stars classified as M- or as cool AGB carbon stars. We have also disqualified luminosity class $\mathrm{V}$ objects, as well as objects that have been classified as planetary nebulae (especially those appearing in the Strasbourg-ESO Catalogue of Galactic Planetary Nebulae - Acker 1992). Post-AGB objects collected in our database include the following classes (which are not mutually exclusive):

- IRAS selected sources - IRASsel in the catalogue. These sources were considered to be post-AGB on the basis of their IRAS colors, found to be between those of AGB stars and those of planetary nebulae (e.g. Kwok et al. 1987; van der Veen et al. 1989; Hu et al. 1993; Garcia-Lario 1997 or; most recently, Suárez et al. 2006). Most of the objects selected in this way are optically faint since the selection criteria focus on the circumstellar material properties. Note, however, that there is a significant overlap between IRAS color-selected, post-AGB sources and other classes of objects, like galaxies, AGB stars, planetary nebulae or young stellar objects (see for example Fig. 3 in Suárez et al. 2006). Therefore, additional observations are usually necessary to classify them properly.

- High Galactic latitude supergiants - hglsg in the catalogue. Massive supergiants are generally not expected to be found at high Galactic latitudes. It is therefore likely that high-latitude supergiants are in fact low-mass stars in their final stages of evolution. Bidelman(1951) was the first to point out the existence of such supergiants (e.g. $89 \mathrm{Her}$ ). However, some of the high-latitude supergiants could also be massive Population I stars that have been ejected from the Galactic disk. Subsequent analyses of chemical abundances confirmed that very often the high-latitude supergiants are

\footnotetext{
2 The exception are non-variable $\mathrm{OH} / \mathrm{IR}$ stars - see below.
}

of low metallicity (in agreement with the interpretation that they are Population II supergiants). The spectral types of such sources are found to be K, G, F and A. In addition, some members of this class of post-AGB objects show extreme Fe-group element depletion (up to -4 dex or even more). Since the abundance of zinc is only mildly weak, the abundance of Fe-group elements cannot reflect the primordial value and a selective removal of metals from the photosphere through grain formation and mass loss has been proposed for those objects (van Winckel et al. 1992). We consider all the very likely post-AGB objects which have $|b|>15^{\circ}$ to belong to the hglsg class (except those classified as $h g l B$ - see below).

- High Galactic latitude B-type supergiants - $h g l B$ in the catalogue and hot post-AGB objects - hotpAGB. Hot, B-type supergiants were discovered in studies of the B-star population in the Galactic halo. Since they are similar to main sequence B-type stars (effective temperature, gravity), detailed chemical composition studies are required to classify these objects properly (e.g. McCausland et al. 1992; Moehler \& Heber 1998, and references therein). We classify as $h g l B$ such very likely post-AGB objects (appropriate chemical composition), which have B spectral type and are located at high Galactic latitudes $\left(|b|>15^{\circ}\right)$. There exists also a group of early-type (A and B) supergiants, located at lower latitudes $\left(|b|<15^{\circ}\right)$, which have been classified as post-AGB on the basis of their chemical composition (see e.g. Parthasarathy et al. 2001; Moehler 2001; Parthasarathy 2004, and references therein). Such sources were included in our catalogue as hotpAGB. Note that some of those sources (the more massive ones?) still display IR excess (e.g. Conlon et al. 1993).

- Bright stars with infrared (IR) excess - IRexc in the catalogue. The IR excess criterion proposed by Parthasarathy \& Pottasch (1986), Pottasch \& Parthasarathy (1988) and others, together with dust mass estimation, is one of the criteria that have been used to select candidate post-AGB sources. However, not all the sources selected in this way can be considered to be post-AGB objects. For example, from the list of 10 infrared excess sources suggested by Pottasch \& Parthasarathy (1988) to be post-AGB objects, we classified five as very likely, one as a possible (we cannot decide if the object is Herbig Ae/Be or post-AGB), and three as disqualified post-AGB objects (Herbig Ae/Be stars). For one of their objects (SAO 253680) there is probably a mistake in terms of its identification in the SIMBAD database. Among the eight objects considered by Hrivnak et al. (1989), on the basis of their infrared excess, as possible post-AGB objects, six appear to be very likely post-AGB and two are disqualified in our catalogue. From the list of 21 post-AGB objects (including RV Tau stars) in Oudmaijer et al. (1992), there are 18 which remain as very likely post-AGB objects in our catalogue while three are disqualified.

- UU Her-type stars - UU Her in the catalogue. Among Population II supergiants, there is a small group of variable stars called UU Her after the best studied member of this group $^{3}$ (Sasselov 1984; Bartkevičius 1992). The characteristic criteria of UU Her-type stars, in addition to the general

\footnotetext{
${ }^{3}$ Note, however, that Klochkova et al. (1997), after a detailed chemical composition analysis of UU Her concluded that this star is a lowmass supergiant but not a post-AGB star. Therefore, UU Her is included in our catalogue as a disqualified post-AGB object, while other members of the group are still classified as UU Her.
} 
hglsg properties, include high radial velocities, small amplitude pulsations (of order of $0.1 \mathrm{mag}$ ) and large IR excess due to circumstellar dust. Bartkevičius (1992) collected the available information on 18 UU Her-type stars, and we classified 12 of them as very likely post-AGB, three as possible post-AGB and three are disqualified in our catalogue. The PS Gem is not in the Bartkevičius (1992) list, but the object is classified in the literature as UU Her-type as well.

- RV Tau stars - RV Tau in the catalogue. The RV Tau stars are highly luminous variable stars, which show alternating deep and shallow minima, periods between 30 and 150 days, and spectral types F, G and K (e.g. Preston et al. 1963). Most of them show IR excess, which has been interpreted by Jura (1986) as evidence of a recent mass loss typical of the AGB phase of evolution. All RV Tauri stars with nearIR excess in their energy distribution (the dusty ones) seem to be binaries, while this is not the case for the naked ones (e.g. Van Winckel et al. 2000). We have considered all the sources classified as RV Tau stars in the SIMBAD database. However, in a few cases we found that they had to be disqualified in the light of the most recent research (e.g. R Sct see Matsura et al. 2002).

- R CrB stars - $\mathrm{R} C r B$; extreme helium stars - eHe; and Late Thermal Pulse objects - LTP in the catalogue. The $\mathrm{R}$ CrB stars are rare $\mathrm{H}$-deficient and $\mathrm{C}$-rich supergiants that undergo irregular declines of up to $8 \mathrm{mag}$ when dust forms in clumps along the line of sight (see e.g. Clayton 1996 for a review). The extreme H-deficiency of the $\mathrm{R} \mathrm{CrB}$ stars suggests that some mechanism removed the entire H-rich stellar envelope. There are two major models which explain their origin: a merger scenario (Webbink 1984; Iben \& Tutukov 1986) and a final helium-shell flash scenario (Fujimoto 1977; Renzini 1979; Iben et al. 1986). There is still no consensus on which of the two scenarios is valid (none of them can explain all the observed properties), but only the second scenario implies a post-AGB nature. We have included $36 \mathrm{R} \mathrm{CrB}$ stars as very likely postAGB objects in our catalogue, but it could be that they are not bona-fide post-AGB stars but simply related objects. Extreme helium stars (16 known with effective temperature below $25000 \mathrm{~K}$ ), which could be evolutionarily connected to R CrB stars (see e.g. Pandey et al. 2001) are also included in our catalogue. In addition we have included two LTP stars (Sakurai's object and FGSge). On the other hand, we did not include V605 Aql, which has experienced a late thermal pulse, but has presently an effective temperature larger than $50000 \mathrm{~K}$.

- 21 micron emission sources - 21 micron in the catalogue. There is a group of 12 sources which show a spectacular emission band at 21 microns (Kwok et al. 1989) confirmed by the Infrared Space Observatory (ISO) observations. All these sources are $\mathrm{C}$-rich and show s-process element enhancement (e.g. Van Winckel \& Reyniers 2000). Since this dust feature is still not well understood we have included information on this class of objects in our catalogue.

- Reflection nebulosity - refneb in the catalogue. Finally, for a few famous objects (Red Rectangle, Minkowski Footprint, Egg Nebula, AFGL 618) we have indicated the characteristic which allowed these sources to be discovered prior to IRAS.

There is one group of objects considered as post-AGB which was intentionally left out of the present edition of the catalogue. These are non-variable OH/IR stars (Habing et al. 1987). Molecular emission in the post-AGB phase of evolution is so
Table 1. The distribution of the very likely post- AGB objects into various classes.

\begin{tabular}{lr}
\hline \hline Class & Number of objects \\
\hline IRASsel & 115 \\
hglsgl & 63 \\
hglB & 9 \\
hotpAGB & 18 \\
IRexc & 34 \\
UU Her & 13 \\
RV Tau & 99 \\
R CrB & 36 \\
eHe & 16 \\
LTP & 2 \\
21 micron & 12 \\
refneb & 4 \\
\hline
\end{tabular}

important and there are so many interesting results that we plan to cover this topic in an upcoming edition of the catalogue. Similarly, the problem of nebular morphology is not covered in the present edition and has been left to the future development of this evolutionary catalogue.

In the light of the above criteria, we have drawn up three lists. The first of these - very likely post-AGB objects - contains 326 sources. An object can belong to this list only if it satisfies at least one of the criteria above and if there is a significant number of references in ADS (at least 5) that do not contradict its post-AGB classification. Using this somewhat arbitrary criterion we considered as possible post-AGB objects those sources that satisfy one of the above criteria but have less than five papers referring to them in ADS. This criterion does not apply in the case of $R V$ Tau, $\mathrm{R} \mathrm{CrB}$ and $e \mathrm{He}$, as there is a consensus on the nature of these objects. We have also classified as possible postAGB those objects for which contradictory opinions appear in the literature and it is not clear which of them is correct. For such cases we have indicated why we cannot treat the object as a very likely post-AGB and have listed the corresponding references. Altogether, we have 107 possible post-AGB sources in our catalogue. The third part of the catalogue contains disqualified objects, i.e. objects that were once considered in the literature as post-AGB objects or candidates, but for which subsequent observations or different interpretations of the data showed that they can no longer be considered as such. This list contains 64 objects. For each of them, we indicate the reason for their disqualification.

The distribution of the very likely post-AGB objects into various classes is shown in Table 1. Note that one object may belong to several classes.

There are 218 objects classified as post-AGB in the SIMBAD catalogue. Of these only 107 are among our very likely postAGB objects, 53 are possible post-AGB, 35 have been disqualified, IRAS 05298-6957 is a LMC object, and we are still keeping 22 objects on the "waiting list" of $\mathrm{OH}$-selected sources as possible non-variable $\mathrm{OH} / \mathrm{IR}$ stars, which are not considered in this edition of the catalogue. There are 99 stars classified as RV Tau in the SIMBAD database. Of these 80 are in our list of very likely post-AGB objects, two are possible, 12 are disqualified and five are not considered in the catalogue since they are LMC sources. On the other hand, we included in the catalogue 19 RV Tauri stars, which are not classified as RV Tau in the SIMBAD database. They are mostly from the recent work of De Ruyter (2006). There are 48 sources classified as R CrB-type stars in the SIMBAD database. Of these 33 belong to our list of very likely Galactic post-AGB sources, two are in the SMC 
and 13 in the LMC. In our catalogue we have, in addition, three sources that are not counted as R CrB by SIMBAD (V3795 Sgr, V348 Sgr and UX Ant). On the other hand, our catalogue contains 66 sources (not classified as $R V$ Tau, $\mathrm{R} \mathrm{CrB}, e \mathrm{He}$ or LTP objects) which are not considered as post-AGB in the SIMBAD database.

Recently, De Ruyter et al.(2006) presented a systematic study of the spectral energy distribution of a sample of 51 postAGB objects and listed the confirmed binary systems. The photospheres of several binary systems also feature the characteristic depletion pattern discussed in the group of hglsg objects. Waters et al.(1992) argued that the most favorable conditions for efficient dust-gas separation occur if the circumstellar dust is trapped in a stable disk configuration. Binary evolution may be responsible for the observed morphology changes in post-AGB objects and in planetary nebulae. (For a review see Sahai 2001; and Balick \& Frank 2002). It is worthwhile pointing out that binarity has a non-negligible role in attracting our attention to such objects, which would have been much less conspicuous otherwise. Therefore, available information about confirmed binarity of post-AGB objects is included in our catalogue together with the corresponding reference listed in the "Bibliography" field (see below). Altogether we have found arguments for the binarity of 47 "very likely" post-AGB objects.

\section{Description of the catalogue}

The on-line version of the catalogue (available at http: //www.ncac.torun.pl/postagb) is organized into 3 sub-catalogues. On the entry screen the user can choose between "very likely post-AGB", "possible post-AGB", and "disqualified objects". When selecting one of the above buttons, the screen indicates the total number of objects of this category in our database and lists them in order of increasing Galactic longitude and latitude $(l, b)$ (Col. 1), the IRAS identification (Col. 2), HD and SAO identifications (Cols. 3 and 4, respectively), other names, if any ${ }^{4}$ (Col. 5), classification(-s) (Col. 6), information about binarity and spectral type ${ }^{5}$ (Cols. 7 and 8 , respectively), and the bibliography related to the object (the ADS link gives connection to ADS with the full listing of bibliography, while the papers we selected can be reached by clicking on the corresponding acronyms). The full list of the selected bibliographic entries can be seen by clicking "Bibliography" on the main bar above the object list. As a rule we did not add selected bibliographic entries when the total number of references for a given object in ADS was less than 10, except for the newest references: De Ruyter et al.(2006) and Suárez et al.(2006), as well as for one object (CD-23 5180) for which we have added a reference related to its binarity. Note, in addition, that there are seven objects classified in SIMBAD as $R V$ Tau (V1899 Sgr, BD+262763, MT Lyr, GLMP 255, V411 Sco, V400 Sco, V558 Sgr), with no bibliographic entries in ADS (SIMBAD). These 7 RV Tauri sources do not have links to ADS in the bibliography field of our catalogue.

4 "other name" is either the usual name or the designation in one of the following main catalogues (in order of preference): General Catalogue of Variable Stars; LS or LSE; BD; CD catalogues and any other name deemed appropriate (if none of the above main catalogues has info about the object).

5 For the vast majority of sources the spectral type is taken from the SIMBAD database. Some exceptions are marked by the bulb icon in our catalogue. Keeping the mouse positioned on the bulb icon for 2-3 s makes a yellow box pop up with more detailed information about the bibliographical source from which we derived the spectral type.
The main bar above the list of objects contains a number of buttons:

- Home - allows the user to get back to the entry screen;

- Change catalogue - allows the selection of one of the subcatalogues;

- Info - show some additional information about the catalogue;

- Search - allows the user to search the sub-catalogue using different criteria (name, class, coordinates, bibliography, etc.);

- Browse - re-opens the full list of objects contained in the selected sub-catalogue;

- Bibliography - provides the full listing of the selected bibliography;

- Export - allows the user to export the required data (see below for details).

When clicking on the $l ; b$ value of a given object (this option exists only for the table of very likely objects) the screen as shown in Fig. 1 appears. The main bar remains the same as on the screen described above. It gives all the characteristics of the object available in the catalogue: the names ${ }^{6}$ and coordinates in degrees as given in the various surveys ${ }^{7}$, the photometric data, and the spectroscopic data: the ISO SWS 01 spectroscopic data (reduced by one of the authors: NS) and the IRAS Low Resolution Spectra (LRS) ${ }^{8}$ taken from the Kevin Volk's Home Page at the University of Calgary http://www.iras. ucalgary.ca/volk/getlrs_plot.html. If the user clicks on the "Finding chart" button, a finding chart is then displayed, generally extracted from 2MASS and from the ESO Digitized Sky Survey-2 Red (see http: //archive. eso.org/dss/dss). When clicking on the "SED" button, the spectral energy distribution appears in [Jy] or in [ $\left.\mathrm{erg} \mathrm{cm}^{-2} \mathrm{~s}^{-1}\right]$, as a function of wavelength, for all the collected photometric data. If ISO SWS 01 and/or IRAS LRS spectra are available, then they are overplotted on the SED diagram in corresponding units.

The data from our catalogue can be exported by clicking on the "Export" button - in the screen shown in Fig. 1. The desired format for the exported table is ascii or Excel.

We plan to follow the literature in order to update the catalogue on a regular basis. The changes introduced will be successively marked in the log file (to be opened when the updating procedure starts). Authors of papers on post-AGB objects are invited to send their comments and/or papers directly to postagb@ncac.torun.pl. However, it will be our responsibility to include new objects in the catalogue, following the precepts given in Sect. 2 .

\section{Some statistical properties of post-AGB objects in our catalogue}

Since the objects appearing in our catalogue have been discovered by various authors and using different selection criteria, there is no well defined selection function. Therefore, our catalogue is not the best suited for population studies. Such studies require well defined samples, e.g. as those obtained by follow-up

\footnotetext{
6 The sign * appearing after an object name means that this counterpart was identified by us and is not shown by SIMBAD.

7 The description of each survey can be obtained by clicking on the corresponding name in the screen shown in Fig. 1.

8 The LRS spectra have the absolute calibration corrected as in Volk \& Cohen (1989) and Cohen et al. (1992).
} 


\section{Post-AGB Object 004.431 -19.448}

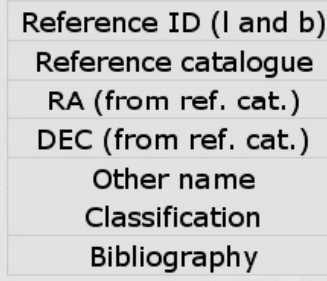

\author{
$004.431-19.448$ \\ 2 mass \\ 289.136498 \\ $-33.522343$ \\ RY Sgr
}

hglsg, IRexc, R CrB

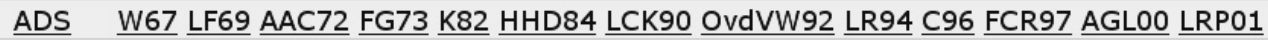

\section{Astrometric data}

\begin{tabular}{|c|c|c|c|c|c|c|c|}
\hline Sky map & \multicolumn{7}{|c|}{ Finding chart from Aladin Sky Atlas } \\
\hline Catalogue & \multicolumn{2}{|c|}{ Object name } & \multicolumn{2}{|c|}{ RA } & \multicolumn{3}{|c|}{ DEC } \\
\hline$\underline{H D}$ & \multicolumn{2}{|c|}{180093} & \multicolumn{2}{|c|}{289.12500} & \multicolumn{3}{|c|}{-33.51667} \\
\hline$\underline{\mathrm{SAO}}$ & \multicolumn{2}{|l|}{211117} & \multicolumn{2}{|c|}{289.13649} & \multicolumn{3}{|c|}{-33.52163} \\
\hline GSC-ACT & \multicolumn{2}{|c|}{0742702655} & \multicolumn{2}{|c|}{289.13658} & \multicolumn{3}{|c|}{-33.52233} \\
\hline GSC2 & \multicolumn{2}{|c|}{ S3322131794* } & \multicolumn{2}{|c|}{289.136525} & \multicolumn{3}{|c|}{-33.522315} \\
\hline TYCHO-2 & \multicolumn{2}{|c|}{742726551} & \multicolumn{2}{|c|}{289.13650306} & \multicolumn{3}{|c|}{-33.52231444} \\
\hline 2MASS & \multirow{2}{*}{\multicolumn{2}{|c|}{$19163275-3331204 *$}} & \multirow{2}{*}{\multicolumn{2}{|c|}{289.136498}} & \multicolumn{3}{|c|}{-33.522343} \\
\hline \multicolumn{4}{|l|}{ DENIS } & & & & \\
\hline \multicolumn{8}{|l|}{$\overline{\mathrm{MS} \times 6 \mathrm{C}}$} \\
\hline IRAS & \multicolumn{2}{|l|}{$19132-3336$} & \multicolumn{2}{|c|}{289.13583} & \multicolumn{3}{|c|}{-33.52194} \\
\hline \multicolumn{8}{|l|}{ Photometric data } \\
\hline SED Diagrams & \multicolumn{3}{|c|}{ SED [Jy] } & \multicolumn{4}{|c|}{ SED [erg $\left.\mathrm{cm}^{-2} \underline{s}^{-1}\right]$} \\
\hline GSC2 magnitude (error) & \multicolumn{3}{|c|}{$B=7.72(0.02)$} & \multicolumn{3}{|c|}{$V=6.9(0.01)$} & $\mathbf{R}$ \\
\hline$\underline{\text { TYCHO-2 }}$ magnitude (error) & \multicolumn{3}{|c|}{$\mathrm{BT}=7.72(0.017)$} & \multicolumn{4}{|c|}{$\mathrm{VT}=6.902(0.011)$} \\
\hline 2MASS magnitude (quality flag) & $\mathrm{J}=5.577(A)$ & & $\mathrm{H}=5$. & $23(A)$ & & $\mathrm{K}=5.139(A)$ & \\
\hline DENIS magnitude (error) & I & & J & & & $\mathrm{K}$ & \\
\hline MSX6C flux [Jansky] (quality flag) & A & & & & & $\mathrm{E}$ & \\
\hline IRAS flux [Jansky] (quality flag) & Fnu12 = $77.2(3)$ & Fnu2 & $26.2(3)$ & Fnu60 $=$ & & Fnu100 $=4.6$ & \\
\hline Spectroscopic data & & & & & & & \\
\hline ISO TDT & & & 495 & 0503 & & & \\
\hline$\underline{\text { IRAS }} \underline{\text { LRS }}$ & & & 1913 & -3336 & & & \\
\hline
\end{tabular}

Fig. 1. An example of the screen shown for a selected object.

studies of well defined candidates (see a colour-selected, fluxlimited sample of IRAS sources described in Suárez et al. 2006). On the other hand, our catalogue can be useful when looking for correlations between various properties of post-AGB objects (e.g. Stasińska et al. 2006; or Siódmiak et al.2007) and when searching for evolutionary connections between different classes of post-AGB objects and their progenitors or progenies.

In Fig. 2 we plot the Galactic longitude distribution for all 326 very likely post-AGB objects (top panel) and for the sample of 272 post-AGB objects with helium stars excluded: i.e. without $\mathrm{R} \mathrm{CrB}$ stars, extreme helium stars and LTP objects (middle panel). Both panels show a similar distribution with a concentration of objects towards the Galactic center and a significant proportion of objects in the range $-60^{\circ} \leq l \leq+60^{\circ}$. The similarity of both diagrams means that the distribution of helium stars, in fact, does not differ from that of the remaining
post-AGB objects. The bottom panel in this figure shows the Galactic longitude distribution for planetary nebulae taken from the Strasbourg catalogue (Acker et al. 1992). The distributions of planetary nebulae and post-AGB objects are clearly different. One reason is that planetary nebulae detected from strong emission lines are easily spotted, even in the Galactic bulge, while this is not possible, in general, for post-AGB objects. On the other hand, low-mass, post-AGB stars will not be able to develop planetary nebulae so there may be more lower-mass objects among post-AGB sources. This is seen in Fig. 3 (the structure of this figure is same as that of Fig. 2), which compares Galactic latitude distributions for post-AGB objects and planetary nebulae. There is a far smaller proportion of "high Galactic latitude" planetary-nebulae than hglsg post-AGB objects, which, statistically speaking, are probably less massive. However, since post-AGB sources are statistically closer than planetary nebulae, 

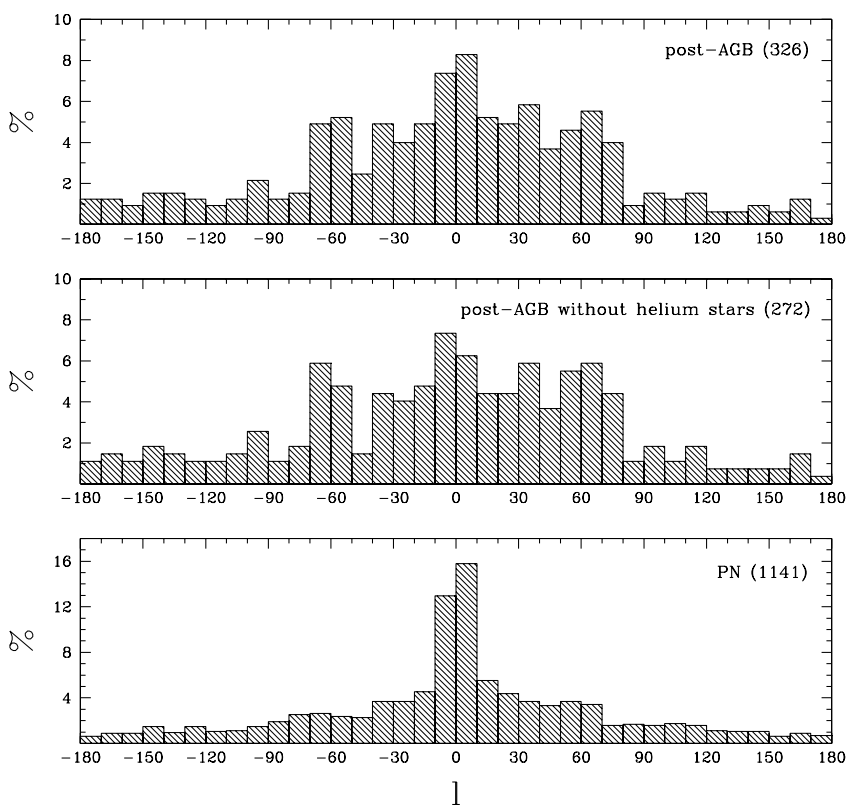

Fig. 2. Galactic longitude distribution for all very likely post-AGB objects from our catalogue (top panel), for post-AGB objects with helium stars excluded: i.e. without $\mathrm{R} \mathrm{CrB}$ stars, extreme helium stars and LTP objects (middle panel), and for planetary nebulae from the Strasbourg catalogue - Acker et al. (1992) (bottom panel). The numbers in parentheses show the number of objects used to make the diagrams.
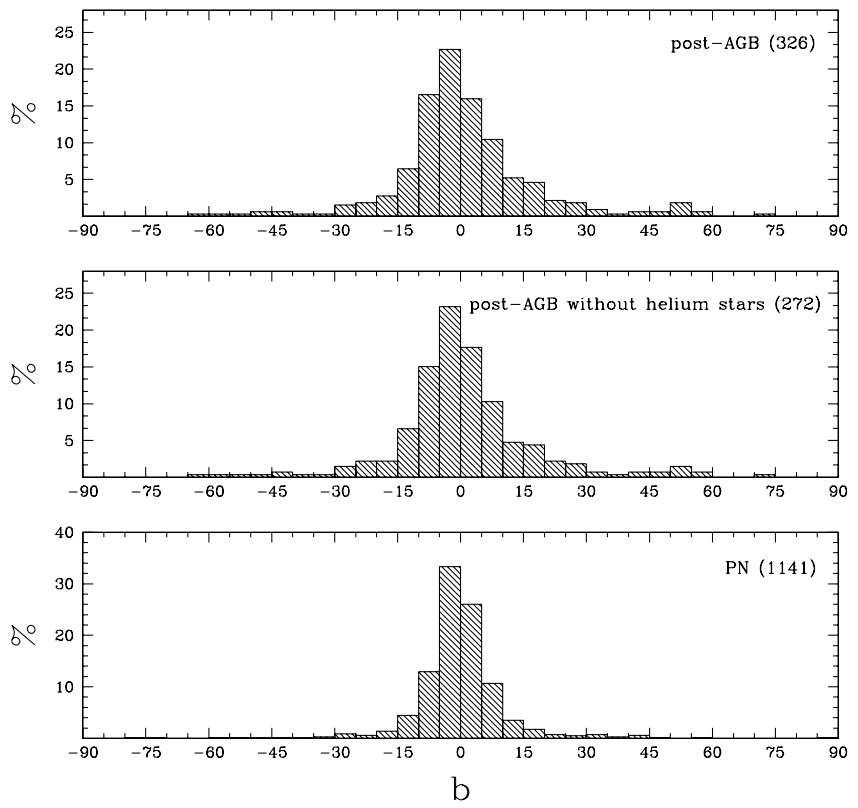

Fig. 3. Galactic latitude distribution for all very likely post-AGB objects from our catalogue (top panel), for post-AGB objects with helium stars excluded: i.e. without $\mathrm{R} \mathrm{CrB}$ stars, extreme helium stars and LTP objects (middle panel), and for planetary nebulae from the Strasbourg catalogue - Acker et al. (1992) (bottom panel). The numbers in parentheses show the number of objects used to make the diagrams.

the proportion of "high Galactic latitude" objects may be relatively larger in the sample of "closer" objects. Note that $y$-scales are the same for the all panels of each figure.

As mentioned before, the IRAS colour-colour diagram was frequently used to search for post-AGB candidates (e.g. Kwok et al.1987; van der Veen et al. 1989; Hu et al. 1993; Garcia-Lario 1997 or; most recently, Suárez et al. 2006). The

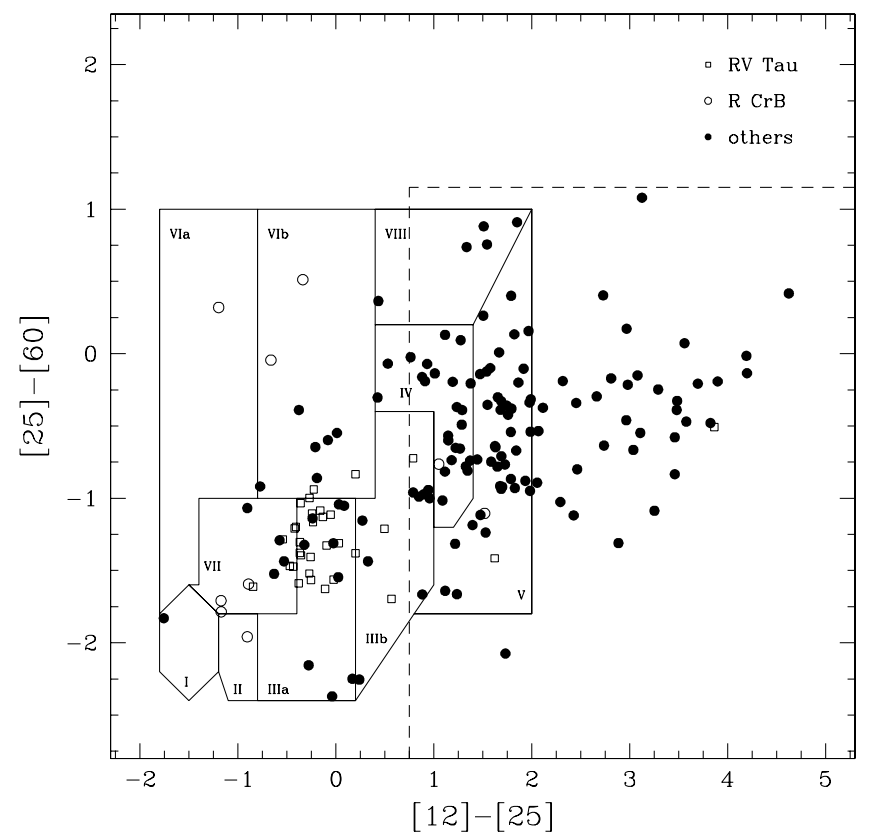

Fig. 4. IRAS colour-colour diagram for 176 very likely post-AGB objects with IRAS flux quality $Q=3$ or 2 at all 3 bands: 12,25 and $60 \mu \mathrm{m}$. RV Tauri stars are marked by open squares, R CrB by open circles, while other objects are represented by filled circles. The regions defined by van der Veen \& Habing (1988) are shown by solid lines, while dashed ones demarcate a typical region of search for post-AGB objects - see text for details.

largest group among very likely post-AGB objects is, in fact, the group of IRASsel sources (115). In Fig. 4 we present IRAS $[25]-[60]=2.5 \log \left[\mathrm{F}_{60} / \mathrm{F}_{25}\right]$ versus [12] $-[25]=2.5 \log \left[\mathrm{F}_{25} / \mathrm{F}_{12}\right]$ diagram for all 176 very likely post-AGB objects with IRAS flux quality, $Q$, equal to 2 or 3 . The zones defined by van der Veen $\&$ Habing (1988) are shown for reference. A typical region of search for post-AGB objects with colors like planetary nebulae is marked by dashed lines (see also Fig. 3 in Suárez et al. 2006) and is defined by [12]-[25] $>0.75$ and [25]-[60] $<1.15$. In Fig. 4 there are 59 very likely post-AGB objects, which are located outside this region. These sources are predominantly RV Tauri and R CrB stars, but not exclusively. It is clear that colour-colour selection, while important, cannot be the only way of selecting post-AGB candidates.

The photometric data that we collected from IRAS and 2MASS catalogues allow us to construct and analyze color-color diagrams based on these data. Figure 5 shows $J-H$ versus $H-K$ (top panel) and $J-K$ versus $K-$ [25] (bottom panel) diagrams for very likely post-AGB objects from our catalogue. The fluxes through the 2MASS filters were transformed to the Johnson system (Carpenter 2000) and the IRAS flux at $25 \mu \mathrm{m}$ was converted to magnitude using [25] $=-2.5 \log \left(\mathrm{F}_{25} / 6.73\right)$ (Beichman et al. 1988). The dotted lines in Fig. 5 separate different groups of objects and are introduced by Siódmiak et al.(2007). DUPLEX sources (as defined by Ueta et al. 2000) have optically thick circumstellar envelopes (they have, therefore, the most red $J-H$ and $J-K$ colors) and are located above the dashed lines (horizontal and inclined, for the top and bottom panel, respectively) in both diagrams. SOLE objects (see Ueta et al.2000) are located below these lines and have $H-K<0.75$ (top panel) and $K-[25]>8$ (bottom panel) as expected for sources with clearly visible central stars and thin dust envelopes. The remaining objects have $H-K>0.75$ (top panel) and $K-[25]<8$ (bottom panel) and are called stellar (no signature of circumstellar 

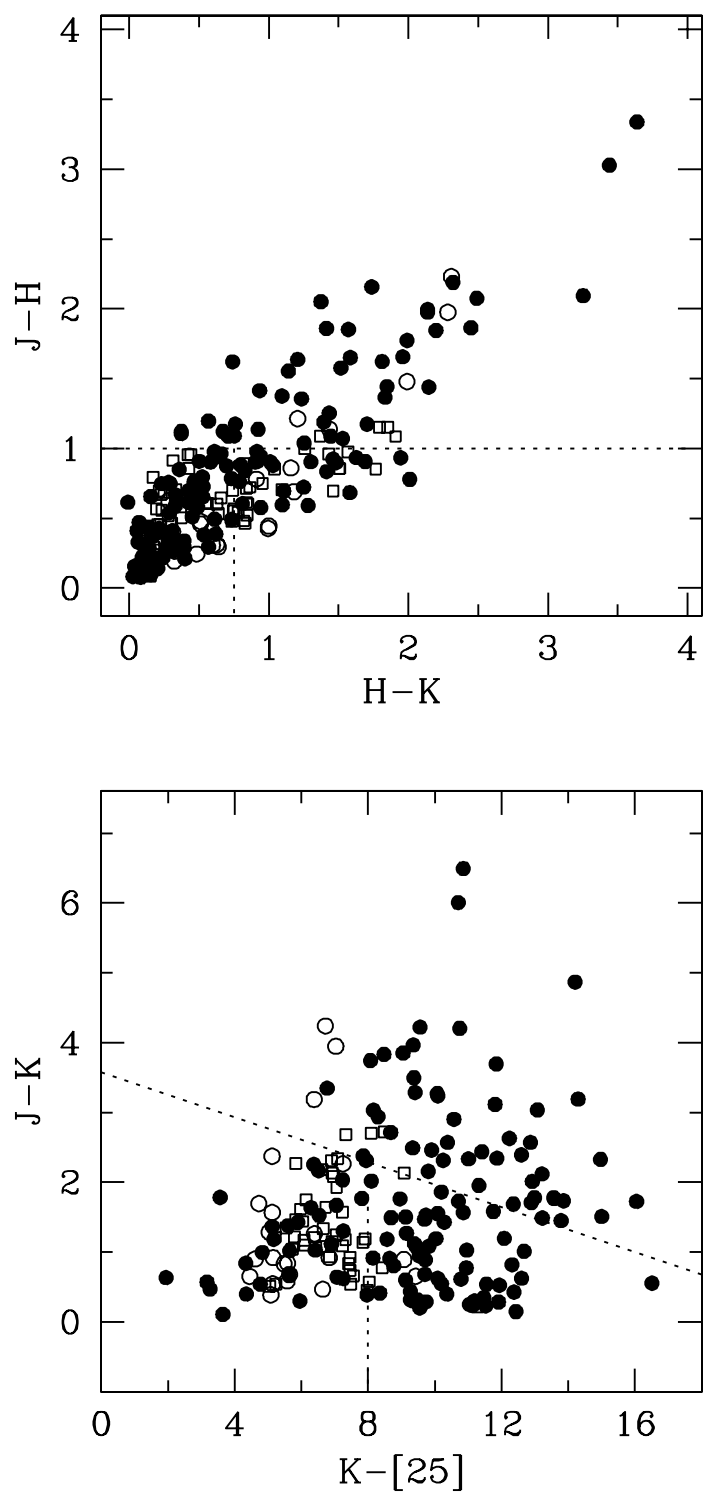

Fig. 5. Infrared color-color diagrams for very likely post-AGB objects from our catalogue. RV Tauri stars are marked by open squares, $\mathrm{R} \mathrm{CrB}$ by opens circles, while other objects by filled circles. Dashed lines separate parts of the diagrams occupied by different group of objects (see Siódmiak et al. 2007).

envelope in the Hubble Space Telescope images) by Siódmiak et al. (2007). As can be seen, most of RV Tauri (open squares) and R CrB stars (open circles) are stellar or SOLE objects, in agreement with their relatively small infrared excess.

Finally, Fig. 6 shows four panels with the distribution of spectral types for the very likely post-AGB objects in our catalogue. The top panels correspond to post-AGB objects located relatively close to the Galactic plane, while the bottom ones correspond to high Galactic latitudes $\left(|b|>15^{\circ}\right)$ sources. The right panels show the spectral type distributions after removing helium stars from the sample.

The most striking feature seen in all four panels is the gap around $10000 \mathrm{~K}$ (spectral type A). The existence of such a gap can be inferred from theoretical models (see e.g. Fig. 6 in Blöcker 1995) and is related to the evolutionary rates during the post-AGB phase of stellar evolution. A similar gap was already observed in the smaller sample of about 220 post-AGB objects discussed by Szczerba et al. (2001) and is also present in the
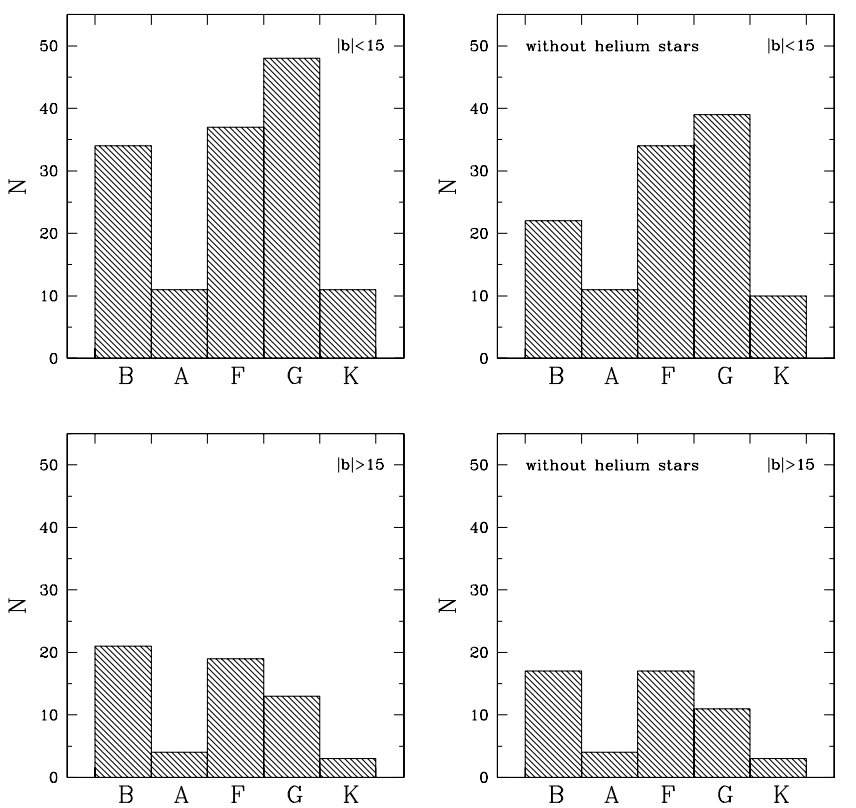

Fig. 6. Distribution of spectral types for very likely post-AGB objects at low (top panels) and high (bottom panels) Galactic latitudes. The right panels show the distribution for post-AGB objects with helium stars excluded:, i.e. without R CrB stars, helium stars and LTP objects.

distribution of spectral types for the colour-selected sample of Suárez et al. (2006). As with the Galactic distribution of postAGB objects discussed above we do not see any significant influence of helium post-AGB stars on the observed distribution of spectral types. This may suggest that the spectral evolution of $\mathrm{H}$-rich and He-rich objects during the post-AGB phase is similar as far as timescales are concerned. On the other hand, it seems that there is a difference in the distribution of spectral types for hglsg and for sources lying closer to the Galactic plane. This is so because there are more F-type than G-type sources among hglsg objects, while the opposite is true for post-AGB objects at lower-Galactic latitudes. This finding seems to contradict theoretically predicted timescales of evolution, i.e. featuring mass loss during the post-AGB phase (Trams et al. 1989). We expect that there are more massive objects among those with lowerGalactic latitudes; however, the massive tracks of Blöcker (1995) predict more F-type stars than G-type ones (see e.g. Fig. 2 in van Hoof et al. 1997). Definitive statements about timescales during the post-AGB phase, based on the statistics of post-AGB objects, require, however, a more complete sample than the one presented in this catalogue together with a proper correction for selection effects.

\section{Summary}

By searching the available literature we were able to identify 326 very likely post-AGB objects in our Galaxy. The collected information was organized into an evolutionary, on-line catalogue of Galactic post-AGB objects, hereafter called the Torun catalogue of Galactic post-AGB and related objects. The present version contains 326 very likely, 107 possible and 64 disqualified postAGB objects. It is anticipated that the number of known postAGB objects will increase significantly in the near future, owing to systematic searches based on infrared surveys. We plan to regularly update our catalogue after new discoveries. The catalogue can be useful when looking for correlations between various properties of post-AGB objects and searching for evolutionary 
connections between different classes of post-AGB objects and their progenitors or progenies. The catalogue has already been successfully used in the work by Stasińska et al. (2006), which showed that post-AGB stars, as a class, are valuable testbeds for theories of AGB nucleosynthesis and in the work by Siódmiak et al. (2007) devoted to the Hubble Space Telescope snapshot survey of post-AGB objects characterized by different physical and chemical properties.

Acknowledgements. This work has been partly supported by grant 2.PN203 019 $31 / 2874$ awarded by the Polish State Committee for Scientific Research and by the European Associated Laboratory "Astronomy Poland-France". We have made extensive use of NASA's Astrophysics Data System Bibliographic Services and SIMBAD database.

\section{References}

Acker, A., Marcout, J., Ochsenbein, F., Stenholm, B., \& Tylenda, R. 1992, Strasbourg - ESO catalogue of Galactic planetary nebulae. Part 1; Part 2

Balick, B., \& Frank, A. 2002, ARA\&A, 40, 439

Bartkevičius, A. 1992, Baltic Astron., 1, 194

Beichman, C. A., Neugebauer, G. Habing, H. J., Clegg, P. E., \& Chester, T. J. 1988, Infrared astronomical satellite (IRAS) catalogs and atlases, Vol. 1, Explanatory Supplement

Bidelman, W. P. 1951, ApJ, 113, 304

Blöcker, T. 1995, A\&A, 299, 755

Carpenter, J. M. 2001, AJ, 121, 2851

Clayton, G. C. 1996, PASP, 108, 225

Cohen, M., Walker, R. G., \& Witteborn, F. C. 1992, AJ, 104, 2030

Conlon, E. S., Dufton, P. L., Keenan, F. P., McCausland, R. J. H., \& Little, J. E. 1993, A\&A, 272, 243

De Ruyter, S., Van Winckel, H., Maas, T., et al. 2006, A\&A, 448, 641

Fujimoto, M. Y. 1977, PASJ, 29, 331

Garcia-Lario, P., Manchado, A., Pych, W., \& Pottasch, S. R. 1997, A\&AS, 126, 479

Geballe, T. R., Tielens, A. G. G. M., Kwok, S., \& Hrivnak, B. J. 1992, ApJ, 387, L89

Habing, H. J., Olofsson, H. 2004, Asymptotic Giant Branch Stars (Springer)

Habing, H. J., van der Veen, \& Geballe, W. 1987, in Late stages of stellar evolution, ASSL, 132, ed. S. Kwok, \& S. R. Pottasch, 91

Hrivnak, B. J., Kwok, S., \& Volk, K. 1989, ApJ, 346, 265

Hu, J. Y., Slijkhuis, S., de Jong, T., \& Jiang, B. W. 1993, A\&AS, 100, 413

Iben, I. Jr, Kaler, J. B., Truran, J. W., \& Renzini, A. 1983, ApJ, 264, 605

Iben, I. Jr, \& Tutukov, A. V. 1985, ApJS, 58, 661

Jura, M. 1986, ApJ, 309, 732

Kleinmann, S. G., Gillett, F. C., \& Joyce, R. R. 1981, ARA\&A, 19, 411

Klochkova, V. G., Panchuk, V. E., \& Chentsov, E. L. 1997, A\&A, 323, 789

Kwok, S. 1993, ARA\&A, 31, 63

Kwok, S., Boreiko, R. T., \& Hrivnak, B. J. 1987, ApJ, 312, 303

Kwok, S., Dopita, M., \& Sutherland, R. 2003, Planetary Nebulae: their evolution and role in the Universe; Astronomical Society of the Pacific, IAU Symp., 209

Kwok, S., \& Pottasch, S. R. 1987, Late stages of stellar evolution (Dordrecht: D. Reidel Publishing Co.), ASSL, 132

Kwok, S., Volk, K., \& Hrivnak, B. J. 1989, ApJ, 345, L51

Le Bertre, T., Lèbre, A., \& Waelkens, C. 1999, Asymptotic Giant Branch stars, IAU Symp., 191

Likkel, L., Morris, M., Omont, A., \& Forveille, T. 1987, A\&A, 173, L11

Lo, K. Y., \& Bechis, K. P. 1976, ApJ, 205, L21
Matsura, M., Yamamura, I., Zijlstra, A. A., \& Bedding, T. R. 2002, A\&A, 387, 1022

McCausland, R. J. H., Conlon, E. S., Dufton, P. L., \& Keenan, F. P. 1992, ApJ, 394,298

Moehler, S. 2001, PASP, 113, 1162

Moehler, S., \& Heber, U. 1998, A\&A, 335, 985

Neugebauer, G., \& Leighton, R. B. 1969, Two-micron sky survey, A preliminary catalogue, NASA SP, Washington: NASA

Oudmaijer, R. D., van der Veen, W. E. C. J., Waters, L. B. F. M., et al. 1992, A\&AS, 96, 625

Paczyński, B. 1971, ApL, 9, 33

Pandey, G., Kameswara, R. N., Lambert, D. L., Jeffery, C. S., \& Asplund, M. 2001, MNRAS, 324, 937

Parthasarathy, M. 2004, in The A-Star Puzzle, ed. J. Zverko, J. Ziznovsky, S. J. Adelman, \& W. W. Weiss (Cambridge, UK: Cambridge University Press), IAU Symp., 224, 379

Parthasarathy, M., \& Pottasch, S. R. 1986, A\&A, 154, L16

Parthasarathy, M., Gauba, G., Fuji, T., \& Nakada, Y. 2001, in Post-AGB objects as a phase of stellar evolution, ed. R. Szczerba, \& S. K. Górny, ASSL, 265, 29

Pottasch, S. R., \& Parthasarathy, M. 1988, A\&A, 192, 182

Preite-Martinez, A. 1987, Planetary and proto-planetary nebulae: From IRAS to ISO (Dordrecht: D. Reidel Publishing Co.), ASSL, 135

Preston, G. E., Krzemiński, W., Smak, J. J., \& Williams, J. A. 1963, ApJ, 137, 401

Renzini, A. 1979, in Stars and Star Systems, ed. B. E. Westerlund (Dordrecht: Reidel), 155

Sahai, R. 2001, in Post-AGB objects as a phase of stellar evolution, ed. R. Szczerba, \& S. K. Górny, ASSL, 265, 53

Sasselov, D. D. 1984, Ap\&SS, 102, 161

Schwarz, H. 1993, Mass Loss on the AGB and Beyond, Second ESO/CTIO Workshop on Mass loss on the AGB and beyond, European Southern Observatory

Schönberner, D. ApJ, 272, 708

Shibai, H. 2004, Adv. Space Res., 34, 589

Siódmiak, N., Meixner, M., Ueta, T., et al. 2006, ApJ, submitted

Stasińska, G., Szczerba, R., Schmidt, M., \& Siódmiak, N. 2006, A\&A, 450, 701

Suárez, O., Garcia-Lario, P., Manchado, A., et al. 2006, A\&A, 458, 173

Szczerba, R., \& Górny, S. K. 2001, Post-AGB objects as a phase of stellar evolution (Dordrech/Boston/London: Kluwer Academic Publishers), ASSL, 265

Szczerba, R., Górny, S. K., \& Zalfresso-Jundziłło 2001, in Post-AGB objects as a phase of stellar evolution, ed. R. Szczerba, \& S. K. Górny, ASSL, 265, 13

Trams, N. R., Waters, L. B. F. M., Waelkens, C., Lamers, H. J. G. L. M., \& van der Veen, W. E. C. J. 1989, A\&A, 218, L1

Ueta, T., Meixner, M., \& Bobrowsky, M. 2000, ApJ, 528, 861

van der Veen, W. E. C. J., \& Habing, H. J. 1988, A\&A, 226, 108

van der Veen, W. E. C. J., Habing, H. J., \& Geballe, T. R. 1989, A\&A, 226, 108

van Hoof, P. A. M., Oudmaijer, R. D., \& Waters, L. B. F. M. 1997, MNRAS, 289,371

Van Winckel, H. 2003, ARA\&A, 41, 391

Van Winckel, H., \& Reyniers, M. 2000, A\&A, 354, 135

Van Winckel, H., Mathis, J. S., \& Waelkens, C. 1992, Nature, 356, 500

Van Winckel, H., Waelkens, C., \& Waters, L. B. F. M. 2000, in The Carbon Star Phenomenon, ed. R. F. Wing (Dordrecht: Kluwer Academic Publishers), IAU Symp., 177, 285

Volk, K., \& Cohen, M. 1989, AJ, 98, 1918

Waelkens, C., \& Waters, L. B. F. M. 2004, in Asymptotic Giant Branch Stars, ed. H. J. Habing, \& H. Olofsson (Springer), 519

Waters, L. B. F. M., Trams, N. R., \& Waelkens, C. 1992, A\&A, 262, L37

Webbink, R. F. 1984, ApJ, 277, 355

Zuckerman, B. 1978, in Proceedings of the Symposium, Planetary Nebulae (Dordrecht: D. Reidel Publishing Co.), 305 